\title{
Flow-driven simulation on variation diameter of counter rotating wind turbines rotor
}

\author{
Y. Fredrika Littik ${ }^{1}$, Y. Heru Irawan $^{2}$, and M. Agung Bramantya ${ }^{3 *}$ \\ ${ }^{1,3}$ Department of Mechanical \& Industrial Engineering, Universitas Gadjah Mada, Yogyakarta, Indonesia \\ ${ }^{2}$ Mechanical Engineering, STTNAS Yogyakarta, Indonesia
}

\begin{abstract}
Wind turbines model in this paper developed from horizontal axis wind turbine propeller with single rotor (HAWT). This research aims to investigating the influence of front rotor diameter variation $\left(\mathrm{D}_{1}\right)$ with rear rotor $\left(\mathrm{D}_{2}\right)$ to the angular velocity optimal $(\omega)$ and tip speed ratio (TSR) on counter rotating wind turbines (CRWT). The method used transient 3D simulation with computational fluid dynamics (CFD) to perform the aerodynamics characteristic of rotor wind turbines. The counter rotating wind turbines (CRWT) is designed with front rotor diameter of $0.23 \mathrm{~m}$ and rear rotor diameter of $0.40 \mathrm{~m}$. In this research, the wind velocity is $4.2 \mathrm{~m} / \mathrm{s}$ and variation ratio between front rotor and rear rotor $\left(\mathrm{D}_{1} / \mathrm{D}_{2}\right)$ are $0.65 ; 0.80 ; 1.20 ; 1.40$; and 1.60 with axial distance $\left(Z / \mathrm{D}_{2}\right) 0.20 \mathrm{~m}$. The result of this research indicated that the variation diameter on front rotor influence the aerodynamics performance of counter rotating wind turbines.
\end{abstract}

\section{Introduction}

Application of wind energy as an alternative energy using wind turbines have been carried out in Indonesia. Wind turbines are used as a power generation system that converts kinetic energy of wind into mechanical energy to drive an electric generator.

Horizontal axis wind turbine propeller type with single rotor is a wind turbine models commonly used in Indonesia. Single rotor wind turbine according to the blade diameter is divided into micro $(\leq 0.1 \mathrm{~m})$, small scale $(0.1 \mathrm{~m}<$ diameter $\leq 1 \mathrm{~m})$, medium scale $(1 \mathrm{~m}<$ diameter $\leq 5 \mathrm{~m})$, and large scale $(>5 \mathrm{~m})$.

The ideal maximum power coefficient of wind turbine dual rotor through actuator disk theory is $64 \%$. Assuming a ratio of the axial velocity on the first rotor is 0.2 and 0.6 on the second rotor. Kumar et al [4], based on the theory of momentum (Betz limit), the maximum energy conversion efficiency of single rotor wind turbine about $59 \%$ while the counter rotating wind turbine with the same swept area increased to $64 \%$.

Various research has been conducted to investigating the influence of diameter rotor using computational fluid dynamics (CFD) aims to analysis aerodynamics performance in wind turbine rotor, both numerically and experimentally demonstrated that ratio of the diameter influenced the performance of counter rotating wind turbine. However, variations in the diameter of the rotor used is still partially in a generally simulation methods. In this research uses the fixed axial distance between front rotor and rear rotor, $\mathrm{k}-\omega$ turbulence models (SST), the solution method Coupled-Scheme and simulation FlowDriven for analyze aerodynamics performance of rotor wind turbine.

\section{Methodology}

\subsection{Computational Fluid Dynamics (CFD)}

Computational fluid dynamics (CFD) used to indicate the numerical solution of the different governing equations of fluid flows. It aims to calculate and analyze a problem that exists. In this case the computer is used as a medium for doing calculations in the form of iteration. CFD consists of three equations set the basis of the calculation process performed computer. The basic principle of a CFD is discretized set into the algebra equations that can be solved by a computer. Equation set in CFD consists of conservation of mass that show in equation (1) and conservation of momentum in equation (2), (3), (4), can be written in Cartesian coordinates as follows:

$\frac{\partial \rho}{\partial t}+\nabla \cdot(\rho \vec{V})$

$\frac{\partial(\rho u)}{\partial t}+\nabla \cdot(\rho u \vec{V})=-\frac{\partial \rho}{\partial x}+\frac{\partial \tau_{x x}}{\partial x}+\frac{\partial \tau_{y x}}{\partial y}+\frac{\partial \tau_{z x}}{\partial z}+\rho f_{x}$

$\frac{\partial(\rho v)}{\partial t}+\nabla \cdot(\rho v \vec{V})=-\frac{\partial \rho}{\partial y}+\frac{\partial \tau_{x y}}{\partial x}+\frac{\partial \tau_{y y}}{\partial y}+\frac{\partial \tau_{z y}}{\partial z}+\rho f_{y}$

$\frac{\partial(\rho w)}{\partial t}+\nabla \cdot(\rho w \vec{V})=-\frac{\partial \rho}{\partial z}+\frac{\partial \tau_{x z}}{\partial x}+\frac{\partial \tau_{y z}}{\partial y}+\frac{\partial \tau_{z z}}{\partial z}+\rho f_{z}$

Where $t$ is the time, $\rho$ is the density of the fluid, $u$ is the velocity of a fluid particle, $\nabla$ is the divergence, $\mathrm{V}$ is volume, $\tau$ is the viscous stress tensor, and $f$ is represents of the inertial forces.

Three phases of the problem analysis in CFD consist of preprocessor, solver, and post-processing. The simulation conducted using $\mathrm{k}-\omega$ turbulence model. This

\footnotetext{
*Corresponding author: bramantya@,ugm.ac.id
} 
model performs well with free shear flows, flat plate boundary layer flows, complicated adverse pressure gradient flows and separated flows.

\subsection{Counter rotating wind turbines}

Lee et al [5], a counter rotating wind turbines (CRWT) is the development of a dual rotor horizontal axle wind turbine, having two rotors rotating in opposite direction on the same axis. The Counter rotating wind turbine model design used in the research can be seen from Table 1 .

The independent variables used in this study are variations on wind speed (u) of $4.2 \mathrm{~m} / \mathrm{s}$ with the variation in the rotor diameter of the front and rotor ratios $\left(\mathrm{D}_{1} / \mathrm{D}_{2}\right)$ $0.65 ; 0.80 ; 1.20 ; 1.40$ and 1.60 . The controlled variable is the ratio of axial distance between two rotors $\left(\mathrm{Z}^{\prime} \mathrm{D}_{1}\right)$ is $0.20 \mathrm{~m}$

Table 1. Properties of counter rotating wind turbines rotor.

\begin{tabular}{|c|c|c|}
\hline Rotor & Front & Rear \\
\hline Diameter $(\mathrm{mm})$ & 230 & 400 \\
\hline Position & Upwind & Upwind \\
\hline Airfoil & NACA 0012 & NACA 0012 \\
\hline Rotation & Clockwise & $\begin{array}{c}\text { Counter } \\
\text { clockwise }\end{array}$ \\
\hline Material & Balsa wood & Balsa wood \\
\hline
\end{tabular}

\subsection{Flow-Driven Simulation}

Flow-driven simulation is a numerical approximation method used by the researcher to calculate the optimal angular velocity of both wind turbine rotor. Calculations are performed every time step until it reaches convergent and stable. This simulation uses the help of a six degree of freedom (6DOF) solver contained in ANSYS Fluent, to determine the direction of rotation of the wind turbine rotor. Using the force and moment imposed in this case the rotor to calculate the translational motion and rotational motion where its center of gravity has been defined.

Equation (5) is an integral form of the conservation equations contained in Flow-Driven simulation:

$\frac{d}{d t} \int_{V} \rho \phi d V+\int_{\partial V} \rho \phi\left(\bar{u}-\bar{u}_{g}\right) \cdot d \bar{A}=\int_{\partial V} \Gamma \nabla \phi \cdot d \bar{A}+\int_{V} S_{\phi} d V$

Where $\rho$ is the fluid density, $\bar{u}$ is the flow velocity vector, $\bar{u}_{g}$ is the mesh velocity of the moving mesh, $\Gamma$ is the diffusion coefficient, and $S_{\phi}$ is the source term of $\phi$.

\subsection{Setup and Solution Solver Simulation}

The general setup used in this 3D simulation is a Transient Pressure-Based type, with turbulence model of SST k- $\omega$ As for the solution method used Coupled-Scheme with second order implicit and time step size 0.01 . For mesh controls use advanced size function on Proximity and curvature with high smoothing sizing mesh. Quality inspection mesh should be taken to ensure no errors during the iterative process, but it also affects the quality of the mesh generated numerical solutions. The steps in this simulation can be seen in the following fig. 1 .
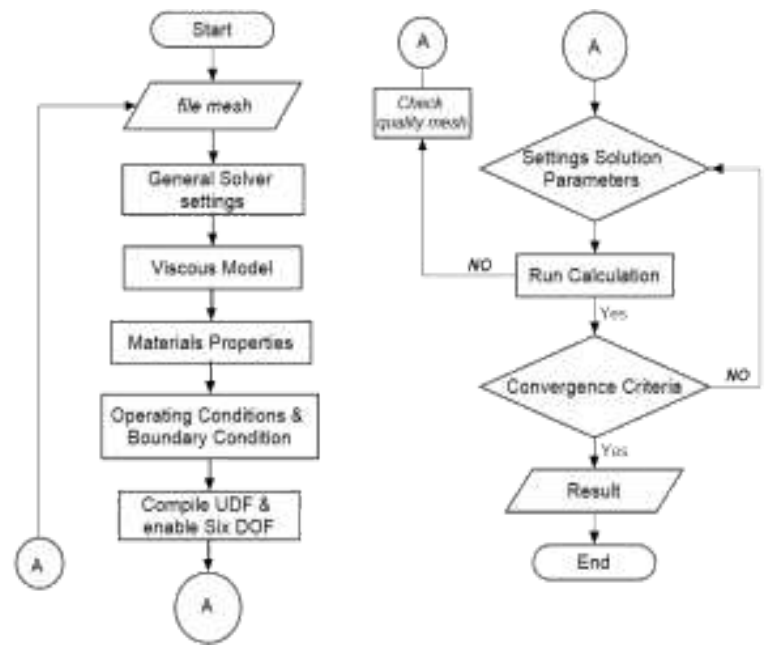

Fig 1. Simulation steps

\section{Result and Discussion}

The 3D simulation results of counter rotating wind turbines show that varying the rotor diameter ratio of front rotor to rear rotor with an incoming wind speed of $4.2 \mathrm{~m} / \mathrm{s}$, has an effect on the aerodynamics performance of the rotors.

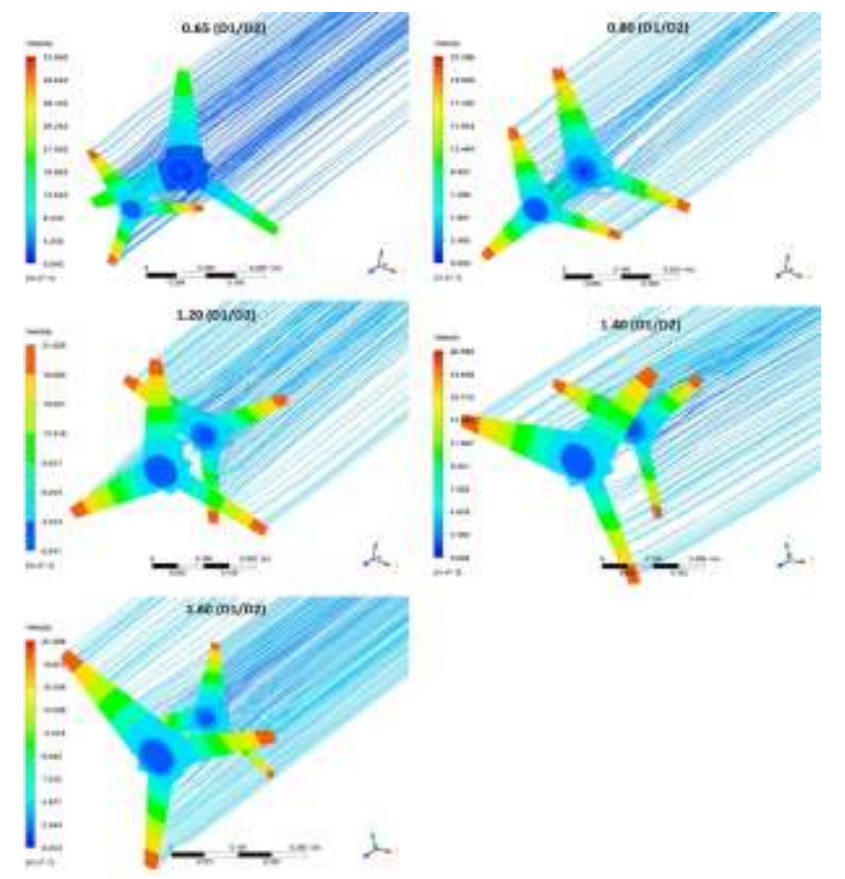

Fig 2. Result of the simulation in $3 \mathrm{D}$ contour of velocity

As shown in fig. 2, the wind velocity that occurs in the front rotor and rotor rear with a diameter ratio of 0.65 increases almost reaching $33.64 \mathrm{~m} / \mathrm{s}$ (front) and $29.44 \mathrm{~m} / \mathrm{s}$ (rear) in stable condition and optimal rotation speed. While the ratio of diameter 0.80 decreased wind speed on both rotor by $19.91 \mathrm{~m} / \mathrm{s}$, the ratio of diameter 1.20 wind velocity that occurred on the rotor increased by $21.03 \mathrm{~m} /$ 
$\mathrm{s}$ due to the larger diameter of the rotor, the ratio of diameters 1.40 and 1.60 has a speed the highest wind is almost the same on both rotor pieces that is equal to 18.64 $\mathrm{m} / \mathrm{s}$ and $18.67 \mathrm{~m} / \mathrm{s}$.

The highest wind speed occurs at the tip of rotor, either on the front or rear rotor at optimum rotation conditions. As for the lowest speed is in the hub of rotor, especially on the rear rotor.

Table 2. Comparison two model wind turbines.

\begin{tabular}{|l|c|c|c|c|}
\hline \multirow{2}{*}{$\begin{array}{c}\text { Wind Turbines } \\
\text { Model }\end{array}$} & \multicolumn{2}{|c|}{ Front Rotor } & \multicolumn{2}{c|}{ Rear Rotor } \\
\cline { 2 - 5 } & $\begin{array}{c}\omega \\
(\mathrm{rad} / \mathrm{s})\end{array}$ & TSR & $\begin{array}{c}\omega \\
(\mathrm{rad} / \mathrm{s})\end{array}$ & TSR \\
\hline SRWT & - & - & 120.30 & 5.73 \\
\hline CRWT (0.65 D1/D2) & 290.62 & 9.00 & 110.03 & 5.24 \\
\hline CRWT (0.80 D1/D2) & 139.71 & 5.32 & 108.18 & 5.15 \\
\hline CRWT (1.20 D1/D2) & 87.29 & 4.99 & 104.91 & 5.00 \\
\hline CRWT (1.40 D1/D2) & 74.74 & 4.98 & 103.70 & 4.94 \\
\hline CRWT (1.60 D1/D2) & 65.53 & 4.99 & 98.06 & 4.67 \\
\hline
\end{tabular}

Table. 2 shows the performance of wind turbines with single rotor and dual rotor. The angular velocity with a single rotor using rotor rear as a comparison with counter rotating wind turbines.

The simulation results obtained by a single rotor wind turbine (SRWT) has an angular velocity of $120.30 \mathrm{rad} / \mathrm{s}$ and TSR 5.73, this is more or less consistent with the assumption (TSR $=5-6$ ) for a three-blade wind turbine. The counter rotating wind turbine with a diameter ratio of 0.65 has an angular velocity of the front rotor of 290.62 $\mathrm{rad} / \mathrm{s}$ and TSR 9, this is because the rotational speed of the rotor is too high. As for the ratio of diameters from 0.80 to 1.60 TSR values ranged from 4 to 5 , this is influenced by decreased angular velocity on the front rotor and rear rotor.

\section{Conclusions}

Counter rotating wind turbines with variations in diameter only the front rotor effect on wind turbine aerodynamic performance, judging by the wind velocity occurring at the rotor end of the front and rear following the change in diameter size from the front rotor to the same axial distance. At a ratio of diameter of 1.20 be it the front rotor and the rear rotor, has a high wind speed at the tip of rotor is $21.03 \mathrm{~m} / \mathrm{s}$. This shows the stability of the rotational speed at this diameter ratio better than the ratio of the other diameter, so also when viewed from the results of angular velocity and its TSR. The greater the number of blades on a wind turbine rotor, the greater the angular velocity and the decreasing TSR value.

\section{References}

1. C.J. Bai, W.C. Wang, Review of Computational and Experimental Approaches to Analysis of Aerodynamic Performance in Horizontal-Axis Wind Turbines (HAWT's), Renewable and Sustainable Energy Reviews, vol. 63, pp. 506-519, (2016)

2. A.D. Hoang, C.J. Yang, Design and Performance Evaluation of a $10 \mathrm{~kW}$ Scale Counter Rotating Wind Turbine Rotor, Journal of The Korean Society of Marine Environment and Safety, vol. 20, pp. 104-112, (2014)

3. S.N. Jung, T.S. No, K.W. Ryu, Aerodynamic Performance Prediction of a $30 \mathrm{~kW}$ Counter Rotating Wind Turbine System, Jeonju : ResearchGate, (2004)

4. P.S. Kumar, A. Abraham, R.J. Bensingh, and S. Ilangoyan, Computational and Experimental Analysis of a Counter Rotating Wind Turbine System, Journal of Scientific and Industrial Research, vol. 72, pp. 300306, (2013)

5. S. Lee, H. Kim, E. Son, S. Lee, Effects of Design Parameters on Aerodynamic Performance of a Counter Rotating Wind Turbine, Renewable Energy, vol. 42, pp.140-144, (2012)

6. M.A. Bramantya, L. Al Huda, An Experimental Study on The Mechanics Power of Counter Rotating Wind Turbines Model Related with Axial Distance Between Two Rotors, 6th International Annual Engineering Seminar (InAES), Yogyakarta, Indonesia, (2016)

7. L. Oggiano, CFD simulations on the NTNU wind turbine rotor and comparison with experiments, Renewable Energy Research Conference, Energy Procedia vol. 58, pp. 111 - 116, (2014)

8. W.Z. Shen, V.A.K Zakkam, J.N. Sorensen, K. Appa, Analysis of Counter-Rotating Wind Turbines, Journal of Physics: Conference Series 75, pp. 012003, (2007)

9. Y.H. Irawan, M.A. Bramatya, Numerical Simulation of The Effect of Axial Distance Between Two Rotors in Counter-Rotating Wind Turbines, $2^{\text {nd }}$ International Conference on Science and Technology Computer (ICST), Yogyakarta, Indonesia, (2016) 